\title{
Sanidade e germinação de sementes de Clitoria fairchildiana tratadas com extratos de plantas
}

José George Ferreira Medeiros ${ }^{1}$, Aderson Costa Araujo Neto ${ }^{2}$, Nivânia Pereira da Costa Menezes ${ }^{1}$, Luciana Cordeiro do Nascimento ${ }^{1}$

${ }^{1}$ Universidade Federal da Paraíba, BR 079, Km 12, CEP 58397-000, Areia, PB, Brasil

2Universidade Estadual do Sudoeste da Bahia, Estrada do Bem Querer, Km 4, CEP 45083-900, Vitória da Conquista, BA, Brasil

"Autor correspondente:

georgemedeiros_jp@hotmail.com

Termos para indexação:

Extratos vegetais

Patologia de sementes

Controle alternativo

Index terms:

Vegetable extracts

Seed pathology

Alternative control

Histórico do artigo:

Recebido em 29/05/2013

Aprovado em 27/11/2013

Publicado em 31/12/2013

doi: 10.4336/2013.pfb.33.76.541
Resumo - O uso de extratos de plantas com propriedades antimicrobianas é uma alternativa ecológica e promissora para substituir a proteção promovida pela aplicação de fungicidas e podem ser utilizados com outras práticas de manejo integrado de doenças. Essa pesquisa avaliou a interferência da aplicação dos extratos de melão-de-são-caetano (Momordica charantia L.) e alamanda (Allamanda blanchetti L.) sobre a micoflora e a germinação de sementes de Clitoria fairchildiana Howard. Essa espécie foi selecionada por apresentar boa produção de sementes e crescimento rápido. Os tratamentos consistiram do controle (sementes não tratadas); fungicida Dicarboximida ( $240 \mathrm{~g} 100$ kg-1), extratos etanólicos de melão-de-são-caetano e alamanda nas concentrações de 10,100, 500 e 1000 ppm. No teste de germinação avaliou-se a porcentagem de germinação, a primeira contagem e o índice de velocidade de germinação. O extrato de melão-de-são-caetano, na concentração de 1000 ppm, reduz significativamente a micoflora associada às sementes de $C$. fairchildiana, favorecendo seu desempenho germinativo. A concentração de 1000 ppm do extrato de alamanda reduz de forma eficiente o crescimento de Rhizopus stolonifer.

\section{Health and germination of Clitoria fairchildiana seeds treated with plant extracts}

\begin{abstract}
The use of plant extracts with antimicrobial properties is a promising and environmentally friendly alternative to substitute the protection promoted by fungicide application and can be used with other practices of integrated disease management. This study evaluated the interference of Allamanda blanchetti L. and Momordica charantia L. extracts application on the mycoflora and germination of Clitoria fairchildiana seeds. The treatments consisted of control (untreated seeds); dicarboximide fungicide ( $240 \mathrm{~g}$ $100 \mathrm{~kg}-1)$ and ethanol extracts of Momordica charantia and Allamanda blanchetti at concentrations of 10, 100, 500 and $1000 \mathrm{ppm}$. The germination test was evaluated by the percentage of germination, first count and germination speed index. The extract of Momordica charantia at concentration of $1000 \mathrm{ppm}$, significantly reduces the mycoflora associated with of Clitoria fairchildiana seeds, favoring their germination performance. The extract of Allamanda blanchetti at concentration of $1000 \mathrm{ppm}$ efficiently reduces the growth of Rhizopus stolonifer.
\end{abstract}




\section{Introdução}

Em consequência da ação antrópica nas formações florestais do Brasil, especialmente em locais populosos ou com intensa atividade agrícola, verifica-se acentuada redução nestas comunidades, tanto em área física quanto em termos de tamanho genético de suas populações (Silva, 2010). O gênero Clitoria contém espécies com diversos empregos, como em programas de reflorestamento (Scalon et al., 2006).

O sombreiro (Clitoria fairchildiana Howard) é empregado na construção civil como divisórias de casas e forros. Pode ser usado para confecção de brinquedos e caixotaria, por possuir madeira moderadamente pesada e de média resistência, além de seu uso na arborização rural e urbana de parques, jardins, estradas, dentre outros, por suas características ornamentais e sombreamento (Guajará et al., 2003). Por apresentar rusticidade e ser de rápido crescimento, tem sido útil nos reflorestamentos heterogêneos destinados à revegetação e recuperação de áreas degradadas (Lorenzi, 2002).

O sistema de produção de mudas de espécies florestais é uma atividade fundamental no processo produtivo. Porém, apresenta uma série de restrições, principalmente de origem sanitária, devido ao grande número de patógenos associados às sementes e, consequentemente, às mudas resultantes (Moreau, 2011). A qualidade sanitária das sementes é um dos mais importantes aspectos relacionados à produção de mudas sadias, pois micro-organismos podem causar anormalidades e lesões nas plântulas, bem como deterioração das sementes (Piveta et al., 2010), sendo que os maiores danos provocados nas sementes durante a germinação são causados por fungos.

A utilização de produtos naturais é uma alternativa para o controle de patógenos associados a sementes, com a vantagem de redução de custos e ausência de impacto ambiental causado pelos agrotóxicos (Girardi et al., 2009). Extratos vegetais têm sido utilizados como métodos alternativos para inibir o desenvolvimento de fungos, a exemplo de extrato aquoso de melão-de-sãocaetano (Momordica charantia L.) (Martins et al., 2009) e alamanda (Allamanda blanchetti A. DC.) (Gomes, 2011).

O teste de germinação é primordial para a análise de sementes florestais (Carvalho \& Nakagawa, 2012), cujo objetivo é obter informações a respeito da qualidade de sementes para semeadura em viveiro, comparação entre lotes de sementes e obtenção de informações a respeito da viabilidade das mesmas (Brasil, 2009).

Desta forma, essa pesquisa avaliou o efeito de diferentes concentrações de extratos naturais de melão-de-são-caetano (Momordica charantia L.) e alamanda (Allamanda cathartica L.) sobre a micoflora e a germinação de sementes de Clitoria fairchildiana Howard.

\section{Material e métodos}

O presente trabalho foi desenvolvido no Laboratório de Fitopatologia do Centro de Ciências Agrárias da Universidade Federal da Paraíba, no Município de Areia, PB. Os frutos de C. fairchildiana foram colhidos em árvores matrizes no mesmo município, no início da deiscência natural. Após a colheita, os frutos foram levados para o laboratório, onde foram postos para secar ao sol, e as sementes foram retiradas manualmente das vagens, sendo eliminadas as chochas ou danificadas por insetos.

O teor de água das sementes foi determinado pelo método da estufa a $105 \pm 3{ }^{\circ} \mathrm{C}$, por $24 \mathrm{~h}$ (Brasil, 2009), utilizando quatro repetições de $5 \mathrm{~g}$ de sementes. Os resultados foram expressos em porcentagem.

Para a obtenção dos extratos de melão-de-são-caetano (M. charantia) e alamanda ( $A$. blanchetti), utilizou-se folhas de plantas nativas coletadas no mesmo município de realização do trabalho.

Os extratos foram preparados pelo método de extração a frio, em que o material vegetal (folhas) foi seco em estufa a $40{ }^{\circ} \mathrm{C}$ durante $72 \mathrm{~h}$ e posteriormente triturado em um moinho de facas para a obtenção do pó vegetal.

Utilizaram-se $150 \mathrm{~g}$ do pó vegetal, imerso em becker contendo $500 \mathrm{~mL}$ de etanol absoluto durante $72 \mathrm{~h} \mathrm{em}$ temperatura ambiente $\left(25 \pm 2{ }^{\circ} \mathrm{C}\right)$, sendo a solução filtrada em papel de filtro. Após este procedimento, o solvente foi extraído com evaporador rotativo por aproximadamente $2 \mathrm{~h}$ a $78^{\circ} \mathrm{C}$ para a obtenção do extrato bruto etanólico (Gomes, 2011).

A partir do extrato bruto, realizou-se a diluição do mesmo nas concentrações utilizadas: 10, 100, 500 e 1000 ppm.

$\mathrm{Na}$ avaliação da sanidade foram utilizadas 200 sementes por tratamento (dez repetições de vinte unidades), as quais foram inicialmente submetidas à assepsia com hipoclorito de sódio (1\%) durante $3 \mathrm{~min}$. 
Os tratamentos consistiram de controle (sementes não tratadas); fungicida dicarboximida (240 g $\left.100 \mathrm{~kg}^{-1}\right)$; extrato de melão-de-são-caetano (EMc) a 10 ppm; EMc a 100 ppm; EMc a 500 ppm; EMc a 1000 ppm; extrato de alamanda (EAb) a 10 ppm; EAb a 100 ppm; EAb a 500 ppm e EAb a 1000 ppm.

A avaliação da incidência de fungos nas sementes foi feita a partir da visualização dos fungos sobre as mesmas através do método de incubação em papel de filtro (Blotter test) (Zauza et al., 2007).

As sementes foram incubadas em placas de Petri sobre uma camada dupla de papel de filtro esterilizado e umedecido com água destilada esterilizada (ADE). As placas contendo as sementes permaneceram durante sete dias a $25 \pm 2{ }^{\circ} \mathrm{C}$. Para a detecção e a identificação dos fungos foi utilizado o microscópio ótico e estereoscópico, sendo comparadas às descrições constantes na literatura (Menezes \& Oliveira, 1993; Marthur \& Kongsdal, 2003). Os resultados foram expressos em porcentagem de incidência de fungos por repetição (Henning, 1994; Goulart, 1997).

No teste de germinação foram utilizadas 200 sementes, divididas em quatro repetições de 50 para cada tratamento, distribuídas em papel, marca Germitest, previamente esterilizado e umedecido com água destilada, na proporção de 2,5 vezes o seu peso seco, e mantidas em equipamento do tipo Biochemical Oxigen Demand (B.O.D) regulado a $25^{\circ} \mathrm{C}$, com fotoperíodo diário de $8 \mathrm{~h}$.

As avaliações foram realizadas aos 9 e aos 15 dias após a semeadura e efetuadas segundo os critérios estabelecidos pelas regras de análise de sementes (Brasil, 2009).

Para estimar o vigor, foram considerados os resultados da primeira contagem do teste de germinação e o cálculo do índice de velocidade de germinação (IVG). A primeira contagem refere-se à quantidade acumulada de plântulas normais obtida aos nove dias após a semeadura e os resultados foram expressos em porcentagem.

Para a determinação do índice de velocidade de germinação (IVG) foram realizadas contagens diárias das plântulas, adotando como critério de avaliação as que continham raiz com $2 \mathrm{~cm}$ de comprimento e calculado através da fórmula proposta por Maguire (1962).

O delineamento utilizado para os testes de sanidade e de germinação foi o inteiramente casualizado e as médias foram comparadas pelo teste de Tukey, ao nível de 5\% de significância. As análises foram realizadas por meio do software estatístico SAS® (SAS INSTITUTE, 1992).

\section{Resultados e discussão}

Os fungos identificados nas sementes de Clitoria fairchildiana (Tabela 1) foram Aspergillus niger, Colletotrichum gloeosporioides, Cladosporium sp., Fusarium oxysporum, Penicillium sp. e Rhizopus stolonifer. Micoflora semelhante foi detectada por Medeiros et al. (2011) avaliando a qualidade sanitária de sementes de flamboyant-mirim (Caesalpinia pulcherrima L.), evidenciando que estes fungos também estão associados à sementes de outras espécies florestais. Fungos dos gêneros Fusarium sp. e Colletotrichum sp. são comprovadamente patógenos de sementes de muitas espécies de plantas (Carvalho \& Muchovej, 1991) e podem ser responsáveis pela variação da germinação das sementes, uma vez que a presença destes fungos compromete consideravelmente o seu desempenho germinativo. Castellani et al. (1996) relatam que sementes predispostas à ação de micro-organismos, quando tratadas, reduzem a capacidade de sobrevivência dos fitopatógenos e potencializam a longevidade das sementes, assim como seu poder germinativo e o vigor das futuras plantas.

O fungicida dicarboximida promoveu redução significativa da incidência dos fungos $C$. gloeosporioides, Cladosporium sp., F. oxysporum, Penicillium sp. e $R$. stolonifer, comprovando a eficiência do tratamento químico para o controle de fungos em sementes (Tabela 1). Machado et al. (2004) utilizando o mesmo produto químico para o tratamento de sementes de outras espécies florestais, a exemplo da caixeta (Tabebuia cassinoides (Lam.) D.C.) e canafístula (Peltophorum dubium (Sprengel) Taubert), comprovaram que o tratamento químico é eficiente para o controle de patógenos em sementes (Mertz et al., 2009). Resultado semelhante foi encontrado por Botelho et al. (2006), comparando a utilização do dicarboximida em sementes de espécies florestais nativas, onde constataram que este fungicida foi o mais eficiente para o controle de fungos em sementes de ipê-amarelo (Tabebuia serratifolia) e ipê-roxo (T. impetiginosa).

A aplicação de extrato de melão-desão-caetano nas concentrações de 500 e 1000 ppm foi eficiente para a redução da incidência de C. gloeosporioides, Cladosporium sp., F. oxysporum e $R$. stolonifer (Tabela 1), enquanto que a concentração de $100 \mathrm{ppm}$ reduziu significativamente a incidência de $A$. niger, demonstrando que o extrato de melão-de-são-caetano apresenta atividade fungitóxica, podendo ser utilizado como alternativa ao controle de patógenos em sementes. 
Tabela 1. Incidência (\%) de fungos em sementes de Clitoria fairchildiana Howard sem tratamento e tratadas com diferentes concentrações de extratos de melão-de-são-caetano (Momordica charantia) e alamanda (Allamanda blanchetti).

\begin{tabular}{ccccccc}
\hline Tratamentos & $\begin{array}{c}\text { Aspergillus } \\
\text { niger }\end{array}$ & $\begin{array}{c}\text { Cladosporium } \\
\text { sp. }\end{array}$ & $\begin{array}{c}\text { Colletotrichum } \\
\text { gloesporioides }\end{array}$ & $\begin{array}{c}\text { Fusarium } \\
\text { oxysporum }\end{array}$ & $\begin{array}{c}\text { Penicillium } \\
\text { sp. }\end{array}$ & $\begin{array}{c}\text { Rhizopus } \\
\text { stolonifer }\end{array}$ \\
\hline Controle & $1,19 \mathrm{ab}$ & $0,99 \mathrm{a}$ & $0,93 \mathrm{a}$ & $1,33 \mathrm{a}$ & $1,35 \mathrm{a}$ & $2,68 \mathrm{a}$ \\
Fungicida & $0,26 \mathrm{~b}$ & $0,3 \mathrm{~b}$ & $0,4 \mathrm{~b}$ & $0,61 \mathrm{~b}$ & $0,51 \mathrm{~b}$ & $1,01 \mathrm{~b}$ \\
EMc $10 \mathrm{ppm}$ & $1,25 \mathrm{ab}$ & $0,49 \mathrm{a}$ & $0,62 \mathrm{ab}$ & $1,01 \mathrm{ab}$ & $0,88 \mathrm{ab}$ & $2,50 \mathrm{a}$ \\
EMc $100 \mathrm{ppm}$ & $0,7 \mathrm{~b}$ & $0,49 \mathrm{a}$ & $0,62 \mathrm{ab}$ & $0,99 \mathrm{ab}$ & $0,88 \mathrm{ab}$ & $2,22 \mathrm{ab}$ \\
EMc $500 \mathrm{ppm}$ & $1,58 \mathrm{a}$ & $0,33 \mathrm{~b}$ & $0,49 \mathrm{~b}$ & $0,6 \mathrm{~b}$ & $0,83 \mathrm{ab}$ & $1,29 \mathrm{~b}$ \\
EMc $1000 \mathrm{ppm}$ & $1,01 \mathrm{ab}$ & $0,3 \mathrm{~b}$ & $0,42 \mathrm{~b}$ & $0,62 \mathrm{~b}$ & $0,59 \mathrm{~b}$ & $1,11 \mathrm{~b}$ \\
EAb 10 ppm & $1,34 \mathrm{ab}$ & $0,7 \mathrm{a}$ & $0,7 \mathrm{a}$ & $0,81 \mathrm{ab}$ & $1,26 \mathrm{a}$ & $2,14 \mathrm{ab}$ \\
EAb 100 ppm & $0,81 \mathrm{ab}$ & $0,7 \mathrm{a}$ & $0,7 \mathrm{a}$ & $0,74 \mathrm{ab}$ & $1,21 \mathrm{a}$ & $1,89 \mathrm{ab}$ \\
EAb 500 ppm & $1,07 \mathrm{ab}$ & $0,7 \mathrm{a}$ & $0,7 \mathrm{a}$ & $0,74 \mathrm{ab}$ & $1,12 \mathrm{ab}$ & $1,55 \mathrm{ab}$ \\
$\mathrm{EAb} 1000 \mathrm{ppm}$ & $1,17 \mathrm{ab}$ & $0,7 \mathrm{a}$ & $0,7 \mathrm{a}$ & $0,74 \mathrm{ab}$ & $1,08 \mathrm{ab}$ & $1,19 \mathrm{~b}$ \\
\hline $\mathrm{CV}(\%)$ & 9,72 & 12,23 & 8,48 & 10,33 & 5,67 & 7,14 \\
\hline
\end{tabular}

Médias seguidas pela mesma letra na coluna não diferem entre si pelo teste de Tukey a 5\%. Dados transformados em $\sqrt{\mathrm{X}+1}$. EMc $=$ extrato de melão-de-sãocaetano; $\mathrm{EAb}=$ extrato de alamanda.

Os fungos dos gêneros Penicillium sp. e Aspergillus sp. são considerados de armazenamento, pois a interação de sementes com esses fungos pode aumentar consideravelmente a velocidade de deterioração das mesmas (Marcos Filho, 2005). Estes patógenos desenvolvem-se rapidamente, levando à redução da viabilidade das sementes, como observado em amendoim bravo (Pterogyne nitens Tul.) (Nascimento et al., 2006).

De acordo com Martins et al. (2009), o extrato de melão-de-são-caetano $(10,20,30$ e $40 \%)$ foi eficiente no controle de fungos em sementes de maniçoba (Manihot glaziovii), porém apresentando variações na redução da incidência entre os diferentes gêneros ou espécies, tais como: Rhizopus sp., Sphaerotheca sp., Aspergillus flavus, Chaetomium globosum e Penicillium sp. De modo semelhante, Leite et al. (2011) destacaram a eficiência do extrato de melão-de-são-caetano para o controle de fungos dos gêneros Fusarium sp. e Cladosporium sp. das sementes de sabiá (Mimosa caesalpiniaefolia Benth.). Dessa forma, a exploração da atividade biológica dos compostos secundários presentes em extratos de plantas medicinais como o melão-de-são-caetano pode representar uma forma potencial de controle alternativo para os fitopatógenos (Swchan-Estrada \& Stangarlin, 2005).

O extrato de alamanda na maior concentração $(1000 \mathrm{ppm})$ reduziu significativamente o crescimento de R. stolonifer. Do mesmo modo, Medeiros et al. (2012), ao utilizar o extrato de alamanda (A. blanchetti) no controle de fungos em sementes de angico (Anadenthera colubrina), observaram uma redução significativa na incidência de Aspergillus sp. e R. stolonifer.

As sementes apresentaram $12 \%$ de umidade. Os resultados de germinação, primeira contagem e índice de velocidade de germinação (IVG) das sementes de C. fairchildiana tratadas com extratos vegetais estão apresentados na Tabela 2.

$\mathrm{O}$ tratamento químico e o extrato de melão-de-sãocaetano na concentração de 1000 ppm proporcionaram aumento significativo na germinação das sementes, comprovando a eficiência desses tratamentos na redução da incidência fúngica (Tabela 1). Estes resultados corroboram com os observados em sementes de zínia (Zinnia elegans), em que o tratamento controle apresentou germinação inferior às sementes tratadas com extratos de alho (Allium sativum) e hortelã (Mentha piperita), devido à alta incidência de patógenos (Girardi et al., 2009).

O percentual de germinação das sementes não tratadas foi similar ao obtido por Lopes et al. (1991) e Castellani et al. (1996) quando observaram que incidência de patógenos associados às sementes pode afetar o parâmetro fisiológico e, em alguns casos, inibir a sua germinação.

A análise da sanidade de sementes utilizando extratos vegetais tem sido avaliada por diversos pesquisadores como Souza et al. (2002), Mieth et al. (2007) e Mata et al. (2009), que demonstraram o efeito positivo dos extratos para a redução na micoflora e o favorecimento da germinação das sementes. 
Tabela 2. Valores médios de germinação, primeira contagem e índice de velocidade de germinação (IVG) de sementes Clitoria fairchildiana Howard sem tratamento e tratadas com extratos de melão-de-são-caetano (Momordica charantia) e alamanda (Allamanda blanchetti).

\begin{tabular}{|c|c|c|c|}
\hline Tratamentos & $\begin{array}{c}\text { Germinação } \\
(\%)\end{array}$ & $\begin{array}{c}\text { Primeira } \\
\text { contagem } \\
(\%)\end{array}$ & IVG \\
\hline Controle & $51 \mathrm{~b}$ & $21 \mathrm{~b}$ & $7,00 \mathrm{a}$ \\
\hline Fungicida & $81 \mathrm{a}$ & $58 \mathrm{a}$ & $4,50 \mathrm{~b}$ \\
\hline EMc 10 ppm & $58 \mathrm{ab}$ & $34 \mathrm{ab}$ & $6,50 \mathrm{ab}$ \\
\hline EMc 100 ppm & $58 \mathrm{ab}$ & $27 \mathrm{~b}$ & $5,00 \mathrm{ab}$ \\
\hline EMc 500 ppm & $60 \mathrm{ab}$ & $42 \mathrm{ab}$ & $5,00 \mathrm{ab}$ \\
\hline EMc 1000 ppm & $79 \mathrm{a}$ & $55 \mathrm{a}$ & $5,00 \mathrm{ab}$ \\
\hline EAb 10 ppm & $53 \mathrm{~b}$ & $23 \mathrm{~b}$ & $7,00 \mathrm{a}$ \\
\hline EAb 100 ppm & $53 \mathrm{~b}$ & $37 \mathrm{ab}$ & $6,70 \mathrm{ab}$ \\
\hline EAb 500 ppm & $53 \mathrm{~b}$ & $37 \mathrm{ab}$ & $6,00 \mathrm{ab}$ \\
\hline EAb 1000 ppm & $57 \mathrm{ab}$ & $48 \mathrm{ab}$ & $6,00 \mathrm{ab}$ \\
\hline CV $(\%)$ & 17,23 & 12,04 & 9,07 \\
\hline
\end{tabular}

Médias seguidas por mesma letra, na coluna, não diferem entre si pelo teste de Tukey ao nível de 5\% probabilidade. EMc = extrato de melão-de-sãocaetano; $\mathrm{EAb}=$ extrato de alamanda.

Os resultados da primeira contagem do teste de germinação indicaram que o tratamento químico e o extrato de melão-de-são-caetano, na concentração de 1000 ppm, superaram os das sementes não tratadas (Tabela 2). A primeira contagem possibilita determinar o vigor das sementes, possibilitando estimar como será a germinação e a qualidade das mudas (Nakagawa, 1994).

Em relação ao índice de velocidade de germinação (IVG), os resultados relacionados à utilização dos produtos naturais testados não influenciaram a velocidade de germinação das sementes de $C$. fairchildiana, não diferindo estatisticamente das sementes não tratadas. No entanto, o tratamento químico promoveu redução significativa no índice de velocidade em relação às sementes do tratamento controle (Tabela 2).

\section{Conclusões}

A concentração de 1000 ppm do extrato de alamanda (Allamanda blanchetti) reduz de forma eficiente o crescimento de Rhizopus stolonifer em sementes de $C$. fairchildiana.

A maior concentração do extrato de melão-de-sãocaetano (1000 ppm) mostrou-se mais eficiente no manejo de todos os fungos detectados na avaliação sanitária das sementes, promovendo reduções significativas na frequência desses fitopatógenos e favorecendo sua germinação. Assim, sugere-se o uso deste extrato para a redução da micoflora associada às sementes de C. fairchildiana, visto que a utilização de produtos naturais em substituição aos agroquímicos geram ganhos ambientais importantes, além de proporcionar custos reduzidos aos produtores de mudas de espécies florestais.

\section{Referências}

BOTELHO, L. S. Fungos associados às sementes de ipê-amarelo, ipê-roxo, aroeira-pimenteira e aroeira-salsa: incidência, efeitos na germinação, transmissão para plântulas e controle. 2006. 115 f. Dissertação (Mestrado em Agronomia), Escola Superior de Agricultura Luiz de Queiroz, Piracicaba, São Paulo.

BRASIL. Ministério da Agricultura, Pecuária e Abastecimento. Secretaria de Defesa Agropecuária. Regras para análise de sementes. Brasília, DF, 2009. 399 p.

CARVALHO, W. L.; MUCHOVEJ, J. J. Fungos associados às sementes de essências florestais. Revista Árvore, Viçosa, MG, v. 15, n. 2, p. 173-178, 1991.

CARVALHO, N. M.; NAKAGAWA, J. Sementes: ciência, tecnologia e produção. Jaboticabal: FUNEP, 2012. 590 p.

CASTEllani, E. D.; SILVA, A.; BARRETO, M.; AGUIAR, I. B. Influência do tratamento químico na população de fungos e na germinação de sementes de Bauhinia variegata L. var variegata. Revista Brasileira de Sementes, Brasília, DF, v. 18, n. 1, p. 41-44, 1996.

GIRARDI, L. B.; LAZAROTTO, M.; MÜLLER, J.; DURIGON, M. R.; MUNIZ, M. F. B.; BLUME, E. Extratos vegetais na qualidade fisiológica e sanitária de sementes de zínia (Zinnia elegans). Revista Brasileira de Agroecologia, Porto Alegre, v. 4, n. 2, p. 897-900, 2009.

GOMES, E. C. S. Extrato de Allamanda blanchetti na indução de fitoalexinas em sorgo e resistência em videira 'superior seedless' contra Uncinula necator. $2011.98 \mathrm{f}$. Tese (Doutorado em Agronomia), Universidade Federal da Paraíba, Areia.

GOULART, A. C. P. Fungos em sementes de soja: detecção e importância. Dourados: EMBRAPA-CPAO, 1997. 58 p. (EMBRAPA-CPAO. Documentos, 11). 
GUAJARÁ, M.; CARVALHO, A. G.; SANTOS, W.; GONÇALVES, K. Aspectos da biologia de Euphalerus clitoriae Burckhardt \& Guajará, 2000 (Hemiptera: Psyllidae) sob condições de campo. Floresta e Ambiente, Rio de Janeiro, v. 10, n. 1, p. 69-75, 2003.

HENNING, A. A. Patologia de sementes. Londrina: EMBRAPACNPS, 1994. 43 p. (EMBRAPA-CNPSO. Documentos, 90).

LEITE, R. P.; MEDEIROS, J. G. F.; NASCIMENTO, L. C. Produtos naturais e seus efeitos sobre a micoflora e fisiologia em sementes de sabiá (Mimosa caesalpiniaefolia Benth.). In: SEABRA, G.; MENDONÇA, I. (Ed.). Educação ambiental: responsabilidade para a conservação da sociobiodiversidade. João Pessoa: Ed. da UFPB, 2011. p. 559-564.

LOPES, J. C.; JARDIM, I. C. C.; SOBREIRA, D. G.; FORDE, G. H. A.; TATAGIBA, J. S. Associação entre germinação, vigor e sanidade em sementes de milho precoce e normal, produzidos na área experimental do Centro Agropecuário da UFES. Informativo ABRATES, Brasília, DF, v. 1, n. 4, p. 55, 1991.

LORENZI, H. Árvores brasileiras: manual de identificação e cultivo de plantas arbóreas nativas do Brasil. Nova Odessa: Plantarum, 2002. v. 1.368 p.

MACHADO, A. A.; MUNIZ, M. F. B.; HOPPE, J. M.; CAMARGO, R. Influencia de diferentes tratamentos de sementes de cedro (Cedrella fissilis Vell.) e cerejeira (Eugenia involucrata DC.) sobre a incidência de fungos de armazenamento. Fitopatologia Brasileira, Brasília, DF, v. 29, n. 2, p. 354, 2004.

MARCOS FILHO, J. Fisiologia de sementes de plantas cultivadas. Piracicaba: FEALQ, 2005. 495 p.

MAGUIRE, J. O. Speed of germination and in selection and evaluation for seedling emergence and vigor. Crop Science, Madison, v. 2, n. 2, p. 176-177, 1962.

MATA, M. F.; ARAÚJO, E.; NASCIMENTO, L. C.; SOUZA, A. E. F.; VIANA, S. Incidência e controle alternativo de patógenos em sementes de mandacaru (Cereus jamacaru DC, Cactaceae). Revista Brasileira de Biociências, Botucatu, v. 7, n. 4, p. 327-334, 2009.

MARTINS, M. T. C. S.; NASCIMENTO, L. C.; ARAÚJO, E. R.; RÊGO, E. R.; BRUNO R. L. A.; FÉLIX, L. P. Atividade antifúngica de extrato de melão-de-são-caetano em sementes de maniçoba. Horticultura Brasileira, Brasília, DF, v. 27, n. 3, S1246-1253, 2009.

MARTHUR, S. B.; KONGSDAL, O. Common laboratory seed health testing methods for detecting fungi. Basserdorf: International Seed Testing Association, 2003.

MEDEIROS, J. G. F.; LEITE, R. P.; NASCIMENTO, L. C. Extratos vegetais e seus efeitos na sanidade e fisiologia de sementes de flamboyant-mirim (Caesalpinia pulcherrima L.). In: SEABRA, G.; MENDONÇA, I. (Ed.). Educação ambiental: responsabilidade para a conservação da sociobiodiversidade. João Pessoa: Ed. da UFPB, 2011. p. 373-377.

MEDEIROS, J. G. F.; NASCIMENTO, L. C.; COSTA, N. P. Controle de fungos em sementes de angico utilizando extratos vegetais. In: CONGRESSO BRASILEIRO DE FITOPATOLOGIA, 45., 2012, Manaus. Resumos... Manaus: Editora Amazônica, 2012. p. 5-9.

MENEZES, M.; OLIVEIRA, S. M. A. Fungos fitopatogênicos. Recife: Imprensa Universitária da UFRPE, 1993. 277 p.
MERTZ, L. M.; HENNING F. A.; ZIMMER P. D. Bioprotetores e fungicidas químicos no tratamento de sementes de soja. Ciência Rural, Santa Maria, RS, v. 39, n. 1, p. 13-18, 2009.

MIETH, A.; PIVETA, G.; PACHECO, C.; HAMANN, F. A.; RODRIGUES, J.; MUNIZ, M. F. B.; BLUME, E. Microflora e qualidade fisiológica de sementes de cedro (Cedrella fissilis) tratadas com extrato natural de hortelã (Mentha piperita). Revista Brasileira de Agroecologia, v. 2, n. 2, p. 1192-1195, 2007.

MOREAU, J. S. Germinação de sementes em diferentes substratos e caracterização morfológica de plântulas de Anadenanthera macrocarpa (Benth.) Brenan. 2011. 45 f. Monografia (Graduação em Agronomia), Universidade Federal do Espírito Santo, Alegre.

NAKAGAWA, J. Testes de vigor baseados na avaliação das plântulas. In: VIEIRA, R. D.; CARVALHO, N. M. Testes de vigor em sementes. Jaboticabal: FUNEP, 1994. p. 49-85.

NASCIMENTO, W. M. O.; CRUZ, E. D.; MORAES, M. H. D.; MENTEN, J. O. Qualidade sanitária e germinação de sementes de Pterogyne nitens Tull. (Leguminosae - Caesalpinioideae). Revista Brasileira de Sementes, Brasília, DF, v. 28, n. 1, p.149-153, 2006.

PIVETA, G.; MENEZES, V. O.; PEDROSO, D. C.; MUNIZ, M. F. B.; BLUME, E.; WIELEWICKI, A. B. Superação de dormência na qualidade de sementes e mudas: influência na produção de Senna multijuga (L. C. Rich.) Irwin \& Barneby. Acta Amazônica, Manaus, AM, v. 40, n. 2, p. 281-288, 2010.

SAS INSTITUTE. SAS/STAT software: changes and enhancements, release 6.07. Cary: Statistical Analysis System Institute, 1992. Chapter 16: The MIXED. (SAS. Technical Report P-229).

SCALON, S. P. Q.; MUSSURY, R. M.; SCALON FILHO, H.; FRANCELINO, C. S. F. Desenvolvimento de mudas de aroeira (Schinus terebinthifolius) e sombreiro (Clitoria fairchildiana) sob condições de sombreamento. Ciência e Agrotecnologia, Lavras, v. 30, n. 1, p. 166-169, 2006.

SCHWAN-ESTRADA, K. R. F.; STANGARLIN, J. R. Extratos e óleos essenciais de plantas medicinais na indução de resistência. In: CAVALCANTI, L. S.; DI PIERO, R. M.; CIA, P.; PASCHOLATI, S. F.; RESENDE, M. L. V.; ROMEIRO, R. S. (Ed.). Indução de resistência em plantas a patógenos e insetos. Piracicaba: FEALQ, 2005. p. $125-138$.

SILVA, L. D. Efeito do isolamento sobre alguns caracteres juvenis em pau-brasil (Caesalpinea echinata Lam). 2010. Dissertação (Mestrado em Agronomia) - Universidade Federal Rural do Rio de Janeiro, Seropédica.

SOUZA M. A. A.; BORGES R. S. O. S.; STARK M. L. M.; SOUZA S. R. Efeito de extratos aquosos, metanólicos e etanólicos de plantas medicinais sobre a germinação de sementes de alface e sobre o desenvolvimento micelial de fungos fitopatogênicos de interesse agrícola. Revista Universidade Rural, Seropédica, v. 22, n. 2, p. 181-185, 2002.

ZAUZA, E. A. V.; ALFENAS, A. C.; MAFIA, R. G. Esterilização, preparo de meios de cultura e fatores associados ao cultivo de fitopatógenos. In: ALFENAS, A. C.; MAFIA, R. G. (Ed.). Métodos em fitopatologia. Viçosa, MG: UFV, 2007. p. 23-51. 